\title{
METing SUSY on the $Z$ peak
}

\author{
G. Barenboim ${ }^{1,2}$, J. Bernabeu ${ }^{1,2}$, V. A. Mitsou $^{2}$, E. Romero ${ }^{2}$, O. Vives ${ }^{1,2, a}$ \\ ${ }^{1}$ Departament de Física Teòrica, Universitat de València, C/ Dr. Moliner 50, 46100 Burjassot, Spain \\ 2 IFIC, Universitat de València-CSIC, Parc Científic U.V., C/ Catedrático José Beltrán 2, 46980 Paterna, Spain
}

Received: 30 November 2015 / Accepted: 18 January 2016 / Published online: 1 February 2016

(C) The Author(s) 2016. This article is published with open access at Springerlink.com

\begin{abstract}
Recently the ATLAS experiment announced a $3 \sigma$ excess at the $Z$-peak consisting of 29 pairs of leptons together with two or more jets, $E_{\mathrm{T}}^{\text {miss }}>225 \mathrm{GeV}$ and $H_{\mathrm{T}}>$ $600 \mathrm{GeV}$, to be compared with $10.6 \pm 3.2$ expected lepton pairs in the Standard Model. No excess outside the Z-peak was observed. By trying to explain this signal with SUSY we find that only relatively light gluinos, $m_{\tilde{g}} \lesssim 1.2 \mathrm{TeV}$, together with a heavy neutralino NLSP of $m_{\tilde{\chi}} \gtrsim 400 \mathrm{GeV}$ decaying predominantly to $Z$-boson plus a light gravitino, such that nearly every gluino produces at least one $Z$-boson in its decay chain, could reproduce the excess. We construct an explicit general gauge mediation model able to reproduce the observed signal overcoming all the experimental limits. Needless to say, more sophisticated models could also reproduce the signal, however, any model would have to exhibit the following features: light gluinos, or heavy particles with a strong production cross section, producing at least one $Z$ boson in its decay chain. The implications of our findings for the Run II at LHC with the scaling on the Z peak, as well as for the direct search of gluinos and other SUSY particles, are pointed out.
\end{abstract}

\section{Introduction}

The discovery by ATLAS [1-3] and CMS [4] experiments at the LHC Collider [5,6] of a new particle with a mass of $125 \mathrm{GeV}[7,8]$ and with the expected properties of a Higgs boson has marked the program of high-energy physics for the next coming years. On one side, it is mandatory to be precise enough in the measurements of the properties of the new scalar particle in order to definitively ascertain its nature as the Higgs boson remnant of the electroweak symmetry breaking mechanism. On the other, its mass is still compatible with the requirements imposed by supersymmetry (SUSY) at the expense of moving the SUSY scale above TeV ener-

\footnotetext{
a e-mail: oscar.vives@uv.es
}

gies. Combined with the current LHC constraints (although model dependent in most cases) from data analyses in the first run of LHC, the search for SUSY effects is becoming more restrictive. Nevertheless, the discovery of SUSY would be such an extraordinary event, not only by itself, but for solving pending fundamental experimental and theoretical problems in particle physics, that an intense well-motivated experimental program to search for SUSY effects is of the highest interest.

Within this scenario, the ATLAS Collaboration has recently presented an intriguing excess at the 3 sigma level of $e^{+} e^{-}$and $\mu^{+} \mu^{-}$pairs just at the $Z$ peak $[9,10]$, accompanied by hadronic activity and missing transverse energy (MET). With an integrated luminosity of $20.3 \mathrm{fb}^{-1}$ at a $p-p$ CM energy of $8 \mathrm{TeV}$, the experiment observes a total of 29 pairs of electrons and muons with an invariant mass compatible with the $Z$-boson mass, with an expected background of $10.6 \pm 3.2$ pairs. No excess over the expected background is observed outside the $Z$ peak. ${ }^{1}$ The question that immediately arises is whether SUSY, or some other extension of the Standard Model (SM), is able to explain that excess of $Z+$ MET events taking into account the current limits on beyond the SM physics. A study in those terms within a SUSY framework is presented in this paper.

As we will show, the observed signal can only be explained if one has a large production cross section of heavy SUSY particles (gluinos or squarks) whose decay chain contains about one $Z$-boson per parent particle. If such an explanation is indeed the answer to the observed excess, our study points out the way to confirm it in the Run II of LHC, as well as cosmological implications, in particular the particle content of dark matter in the universe. The resulting scheme of SUSY particle mass hierarchy, including charginos and neutralinos, will be apparent.

\footnotetext{
${ }^{1}$ A similar analysis on $Z$ plus $E_{\mathrm{T}}^{\text {miss }}$ has been performed by CMS [11]. However, among other differences, no cut on $H_{\mathrm{T}}$ has been applied. No deviation from SM expectations has been observed here.
} 


\section{ATLAS excess in $l^{+} l^{-}$on the $Z$ peak}

In order to motivate and clarify the assumptions that will be made in the next sections, a simplified summary of the mentioned ATLAS search is given here.

The main focus of the search in [9] is the decays of squarks and gluinos with two leptons (electrons or muons), jets and $E_{\mathrm{T}}^{\text {miss }}$ in the final state, where the two leptons originate from a $Z$-boson.

In order to discriminate between SM background events and a possible signal, the following requirements are applied:

- At least two same-flavored leptons with opposite electric charge are required in each event. If more than two leptons are present in the event, the two with the largest values of $p_{\mathrm{T}}$ are selected. The leading lepton, i.e. the lepton with highest $p_{\mathrm{T}}$, must have $p_{\mathrm{T}}>25 \mathrm{GeV}$, whereas the subleading lepton $p_{\mathrm{T}}$ can be as low as $10 \mathrm{GeV}$. Their invariant mass must fall within the $Z$-boson mass window, here considered as $81<m_{l l}<101 \mathrm{GeV}$.

- All events are further required to contain at least two jets with $p_{\mathrm{T}}>35 \mathrm{GeV}$ and $|\eta|<2.5$, to have $E_{\mathrm{T}}^{\text {miss }}>225$ $\mathrm{GeV}$ and $H_{\mathrm{T}}>600 \mathrm{GeV}$, where $H_{\mathrm{T}}$ is the $p_{\mathrm{T}}$ sum over all the jets with $p_{\mathrm{T}}>35 \mathrm{GeV}$ and $|\eta|<2.5$ and the two leading leptons: $H_{\mathrm{T}}=\sum_{i} p_{\mathrm{T}}^{\text {jet }, i}+p_{\mathrm{T}}^{\text {lepton, } 1}+p_{\mathrm{T}}^{\text {lepton,2 }}$.

- Furthermore, the azimuthal angle between each of the two leading jets and $E_{\mathrm{T}}^{\mathrm{miss}}$ is required to be higher than 0.4 .

A great effort has been made to accurately estimate the number of SM events that survive the previous selection. An enumeration of these expected SM processes together with some of their characteristics follows:

- The main background, namely $t \bar{t}$, together with $W W$, $W t$, and $Z \rightarrow \tau \tau$, which add up to $\sim 60 \%$ of the predicted background, have been estimated using a datadriven method that has been thoroughly cross-checked with different techniques.

- Diboson backgrounds with real $Z$-boson production $(\sim 25 \%)$ and "rare top" $(t \bar{t}+W, t \bar{t}+Z, t \bar{t}+W W$ and $t+Z)$ backgrounds $(<5 \%)$ are estimated using MC simulation. These are subject to carefully assessed theoretical and experimental uncertainties.

- Processes with "fake leptons", i.e. leptons originating from the mis-identification of a jet, $(\sim 10 \%)$ are estimated using a data-driven method used regularly in most of ATLAS analyses.

- Finally, particular care has been taken to suppress the $Z / \gamma^{*}+$ jets background as much as possible, given that it could mimic a possible signal (the cut in the azimuthal angle between each of the two leading jets and the $E_{\mathrm{T}}^{\text {miss }}$ has been applied to serve this purpose). Nonetheless, a data-driven technique has been used to estimate this small $(<1 \%)$ but important background.

The total number of expected SM model events passing all the requirements is $10.6 \pm 3.2$ and the number of observed data events is 29 . This corresponds to a $3.0 \sigma$ significance.

\section{$3 Z$-boson production in the MSSM}

As explained above, if the observed excess is confirmed, it would clearly point to a new non-standard process producing additional $Z$-bosons at LHC energies. $Z$-bosons are regularly produced in the decay chains of most of the SM extensions. Still, this signal would require a significant production of $Z$-bosons without conflicting with all other experimental searches of beyond the SM particles. In fact, using the central value for the expected background and taking into account the $Z$-branching ratio to muon and electron, this would imply that we have produced $273 \pm 48$ additional $Z$-bosons (with $20.3 \mathrm{fb}^{-1}$ ). Assuming the $Z$-bosons are produced in the decay chains of beyond the SM particles, $Y$, produced in the collision, we need to produce at least $273 / \mathcal{N}(Y \rightarrow Z) Y$ particles, with $\mathcal{N}(Y \rightarrow Z)$ the average number of $Z$-bosons produced in the decay of a $Y$ particle. On the other hand, as we will see next, the experimental cuts used in the experiment, namely $n_{\text {jets }} \geq 2, E_{\mathrm{T}}^{\text {miss }}>225 \mathrm{GeV}$ and $H_{\mathrm{T}}>600 \mathrm{GeV}$, define further the characteristics of the $Y$ particle and its decays.

Now, the question is whether it is possible to produce these extra $Z$-bosons with the associated event attributes in some SUSY extension of the SM, while at the same time all the constraints imposed by present new-physics searches at LHC and other experiments are satisfied. In this section, we will assume that the acceptance of the applied selection, also taking into account the reconstruction efficiencies is ideally equal to unity. In Sect. 4, we will perform a "realistic" simulation in a SUSY model using Delphes [12] to take into account the signal acceptance and detector efficiencies.

\subsection{Production cross section of MSSM particles}

We need to produce 273 (225 at $1 \sigma$ ) $Z$-bosons if we want to accommodate the observed excess in lepton pairs. Assuming that $R$-parity is conserved, supersymmetric particles are produced in pairs in processes of the type $p p \rightarrow Y \bar{Y}$. Thus, the required cross section for this process would be,

$$
\begin{aligned}
& \sigma(p p \rightarrow Y \bar{Y})=\frac{N_{\mathrm{ev}} / \mathcal{N}(Y \rightarrow Z)}{\mathcal{L}} \\
& \quad=\frac{137(113) / \mathcal{N}(Y \rightarrow Z)}{20.3 \mathrm{fb}^{-1}}=\frac{6.7(5.6) \mathrm{fb}}{\mathcal{N}(Y \rightarrow Z)},
\end{aligned}
$$




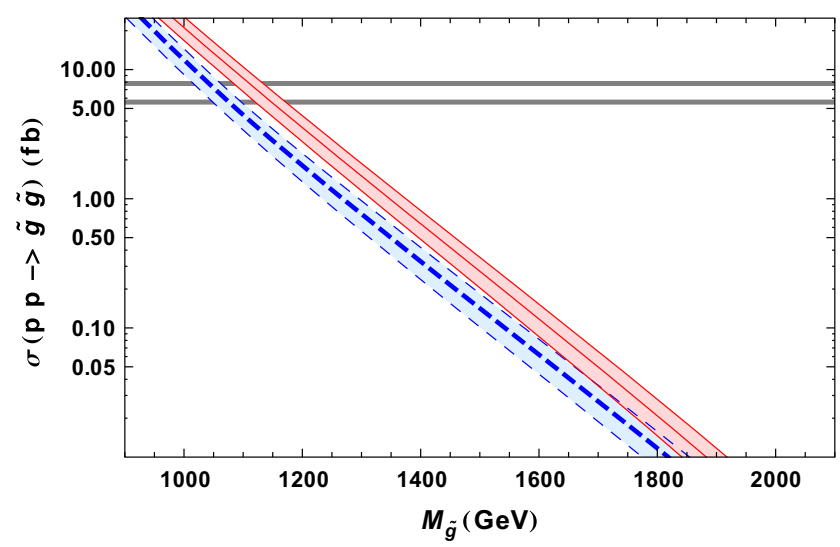

Fig. 1 On the left, we show the production cross section of gluino pairs as a function of the gluino mass for two fixed values of the first generation squark masses: $1000 \mathrm{GeV}$ (blue/dashed) and decou-

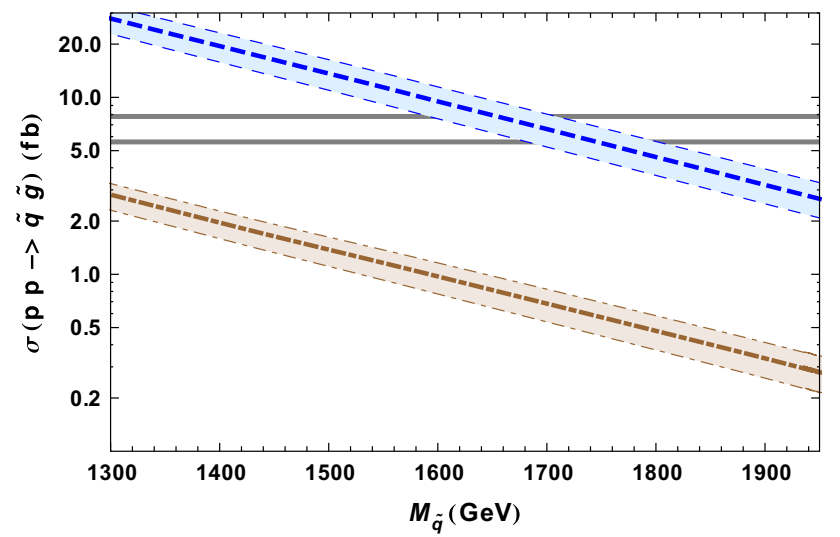

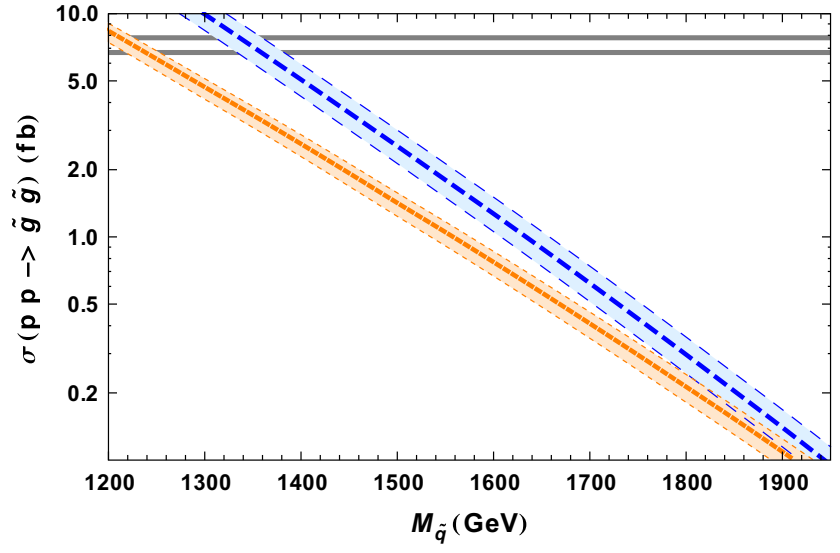

pled squarks ( red/solid). On the right, we have the production cross section of squarks pairs as a function of their mass with gluino masses of $1000 \mathrm{GeV}$ (blue/dashed) and $2500 \mathrm{GeV}$ (orange/dotted)

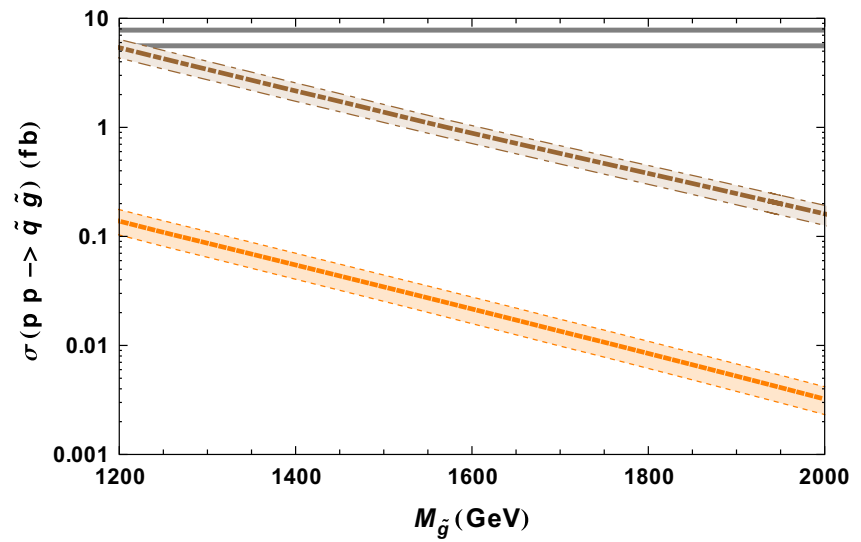

Fig. 2 Production cross section of squark-gluino as a function of the squark mass (left) or the gluino mass (right) for fixed values of the associate particle mass: $1000 \mathrm{GeV}$ (blue/dashed) $1500 \mathrm{GeV}$ (brown/dash-dotted) and $2500 \mathrm{GeV}$ (orange/dotted)

where we take into account that two $Y$ particles are produced in each event. So, if we obtained one $Z$-boson for each $Y$ particle produced, we would need a production cross section of $(6.7 \pm 1.1) \mathrm{fb}$ at the LHC with a CM energy of $8 \mathrm{TeV}$. Then the first thing we must do is to determine whether it is still possible to have these production cross sections for some supersymmetric particle taking into account the constraints from direct searches at LHC. Here, we consider the production cross sections of different supersymmetric particles separately to identify the relevant processes. However, in a full simulation, as done in Sect. 4, different sparticles contribute to the final $Z$ plus jets plus MET signal and the different contributions must be added.

Naively, the first option to consider in a hadron collider would be strong production of squarks or gluinos (assuming they produce $Z$ bosons in their decays). However, current experimental searches of jets plus missing energy at LHC force the masses of these colored particles to be high [1315]. Nevertheless, as we will see below, in some cases we can still find cross sections of the required size.
Production cross sections of gluinos and squarks depend only on their masses and are basically independent of other MSSM parameters. In the case of gluino and squarks of the first generation, the cross section depends both on the squark and gluino masses due to $t$-channel contributions, but in the case of stops or sbottoms it depends only on the stop or sbottom mass. In Fig. 1 we present the production cross section of gluino pairs and light-flavor squark pairs ${ }^{2}$ calculated at NLL+NLO with NLL-fast [16-22] as a function of the gluino or squark mass. Figure 2 shows the squark plus gluino cross section as a function of the squark or gluino mass with the second mass fixed at different values. The different bands in these figures correspond to the cross section at $1 \sigma$ with fixed squark or gluino masses: the blue (dashed) band corresponds to $m_{\tilde{q}, \tilde{g}}=1000 \mathrm{GeV}$, the brown (dashdotted) band to $m_{\tilde{q}, \tilde{g}}=1500 \mathrm{GeV}$, the orange (dotted) band has $m_{\tilde{q}, \tilde{g}}=2500 \mathrm{GeV}$ and the red (solid) band corresponds

\footnotetext{
2 These squark cross sections are obtained with five squark flavors (mass degenerate), i.e. include contributions from sbottoms which are treated as a light flavor.
} 
to decoupled squarks or gluinos. In all these plots, the horizontal gray lines represent the required cross section at $1 \sigma$ needed to reproduce the referred ATLAS excess.

As we can see in these figures, the required cross section is reached only for light gluino and squark masses. In the case of gluino production, the needed cross section is obtained only for $m_{\tilde{g}} \lesssim 1200 \mathrm{GeV}$ and favors heavy squark masses. In fact, these gluino masses are in the boundary of the allowed region obtained from jets plus missing $E_{T}$ searches at LHC [13-15] and would contribute significantly only if every $\tilde{g}$ produces at least a $Z$-boson in its decay. For the production of squark pairs, present limits are $m_{\tilde{q}} \gtrsim 1400 \mathrm{GeV}$ for heavy gluinos and $m_{\tilde{q}} \gtrsim 1650 \mathrm{GeV}$ for degenerate squarks and gluinos [13$15]$. Under these conditions, $\sigma(p p \rightarrow \tilde{q} \tilde{q})$ is always well below the required cross section, even for $m_{\tilde{q}} \gtrsim 1400 \mathrm{GeV}$. Here, we do not show the cross section $\sigma\left(p p \rightarrow \tilde{q} \tilde{q}^{*}\right)$ as it is typically one order of magnitude smaller than $\sigma(p p \rightarrow \tilde{q} \tilde{q})$, and thus irrelevant.

Another important process is the simultaneous production of squark and gluino shown in Fig. 2. However, we see that we would need both squark and gluino to be light, which is not possible if we take into account the bounds from LHC searches [13-15]. In summary, the best option seems to be gluino pair production with $m_{\tilde{g}} \lesssim 1200 \mathrm{GeV}$ with relatively heavy squarks $m_{\tilde{q}} \gtrsim 3000 \mathrm{GeV}$, if we can get at least one $Z$-boson in every gluino decay.

Yet, we can also consider the strong production of stop pairs, where the current bounds on stop masses are much lower, $m_{\tilde{t}} \gtrsim 650 \mathrm{GeV}[23,24]$. The total $\tilde{t} \tilde{t}^{*}$ production cross section is shown in Fig. 3, calculated at NLL+NLO with NLL-fast. In this case, we can see that we could reach the required cross section for $m_{\tilde{t}} \lesssim 750 \mathrm{GeV}$ which in principle could be achievable in general SUSY models (always assuming that every stop produces a $Z$-boson in its decay). However, the cuts $H_{T}>600 \mathrm{GeV}$ and $E_{T}^{\text {miss }}>225$ are very restrictive. We can see this in Fig. 4, where we

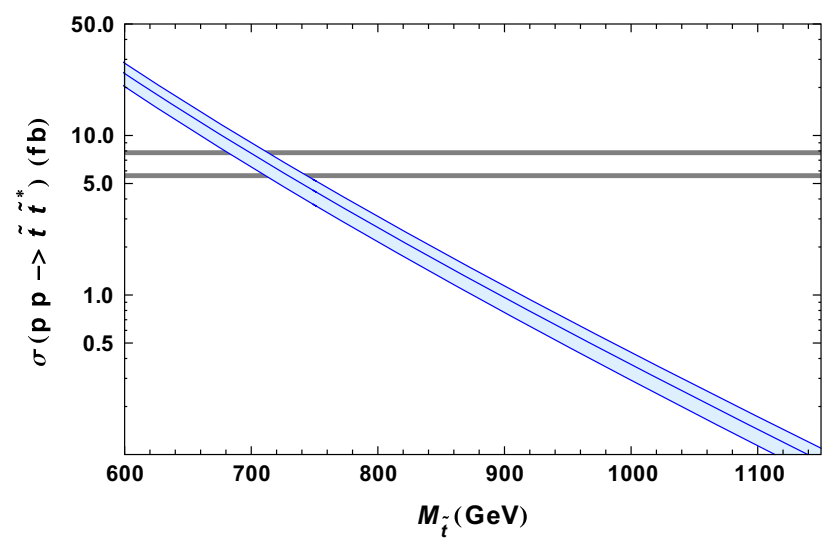

Fig. 3 Production cross section of $\tilde{t} \tilde{t}^{*}$ pairs as a function of the stop mass. The cross section is basically independent of other SUSY masses

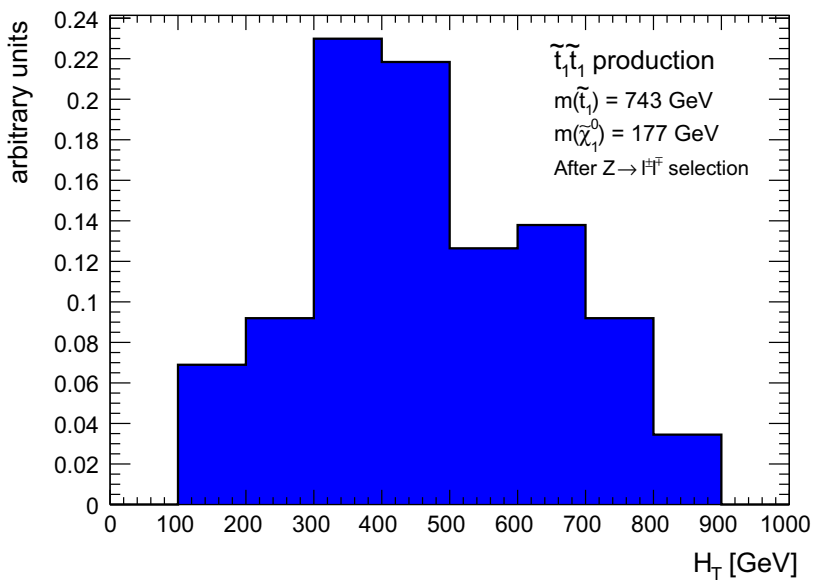

Fig. $4 H_{\mathrm{T}}$ distribution in arbitrary units from the decay of a pair of stops of $m_{\tilde{t}}=743 \mathrm{GeV}$ to jets plus $E_{\mathrm{T}}^{\text {miss }}$ after applying the selection $Z \rightarrow l^{+} l^{-}$

show the $H_{\mathrm{T}}$ distribution from the decays of stop pairs with $m_{\tilde{t}_{1}}=750 \mathrm{GeV}$. As can be seen here, the $H_{\mathrm{T}}$ distribution peaks at $H_{\mathrm{T}} \simeq 400-500 \mathrm{GeV}$ as a consequence of the relatively small stop mass, and only a small fraction of the events are able to overcome the cut on $H_{\mathrm{T}}$. Therefore, we must conclude that it is not possible to generate the required cross section and fulfill the requirements of the observed excess through stop production.

Apart from the production cross sections of colored sparticles, we could still consider the weak production of charginos/neutralinos which can be large enough for light gauginos. Taking into account that the current bounds on chargino and neutralino masses are not very stringent, electroweak production is worth exploring [25,26]. It is well known that the largest electroweak production cross sections are those corresponding to $\tilde{W}^{0} \tilde{W}^{ \pm}$and $\tilde{W}^{+} \tilde{W}^{-}$, in terms of gauge eigenstates. This would correspond to $\chi_{2}^{0} \chi_{1}^{ \pm}$and $\chi_{1}^{+} \chi_{1}^{-}$in terms of mass eigenstates. However, the observed signal requires the $Z$-boson to be on-shell, at least two jets, $E_{T}^{\text {miss }}>225 \mathrm{GeV}$, and a minimum $H_{T}$ of $600 \mathrm{GeV}$. As an example, we present, in Fig. 5, the production cross section $\sigma\left(p p \rightarrow \chi_{1}^{+} \chi_{2}^{0} \rightarrow\left(\chi_{1}^{0} W^{+}\right)\left(\chi_{1}^{0} Z\right) \rightarrow\left(\chi_{1}^{0} j j\right)\left(\chi_{1}^{0} Z^{0}\right)\right)$ in the case where the only restriction imposed is a minimum $p_{T} \geq 20 \mathrm{GeV}$ for the jets (in blue/dashed), and the same cross section after applying a cut on the hadronic $H_{T}^{\text {jets }} \geq 300 \mathrm{GeV}$. As can be seen, the cross section with charginos of $m_{\chi_{1}^{+}} \lesssim 350 \mathrm{GeV}$ looks, in principle, able to accommodate the required $Z$ production. However, the situation changes after we impose the experimental cuts used in the analysis. The required $E_{T}^{\text {miss }}$ is easily obtained if $m_{\chi_{1}^{0}} \gtrsim 150 \mathrm{GeV}$, but the requirement on $H_{T}$ is very restrictive. In fact, for chargino masses $m_{\chi_{1}^{ \pm}} \simeq 350 \mathrm{GeV}$, the largest contribution to the production cross section would correspond to $\sqrt{\hat{s}} \simeq 700 \mathrm{GeV}$, which can never produce $H_{T}$ of $600 \mathrm{GeV}$ and $E_{T}^{\text {miss }}>225 \mathrm{GeV}$. Then we can expect the cross section $\sigma\left(p p \rightarrow \chi_{1}^{0} \chi^{+}\right)$at larger $\sqrt{\hat{s}}$ values to be strongly 


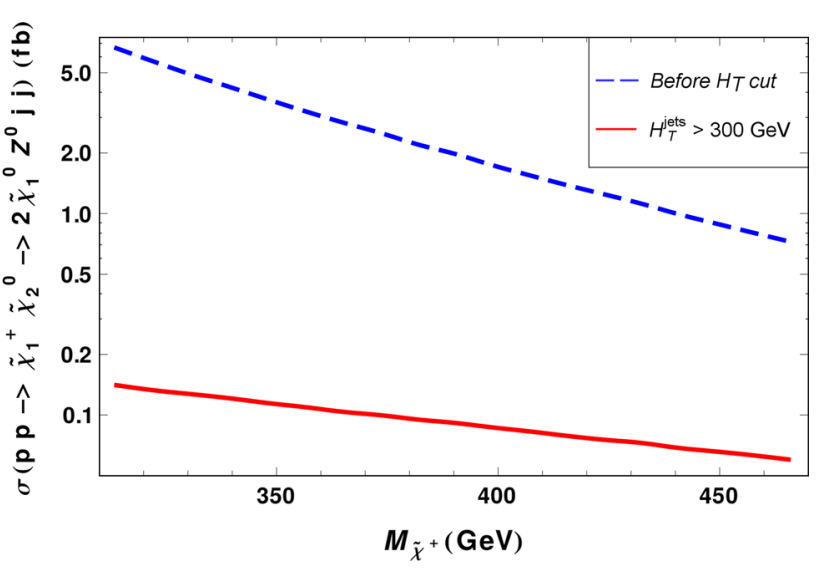

Fig. 5 Production cross section of a pair $\chi_{2}^{0} \chi_{1}^{ \pm}$as a function of $m_{\tilde{\chi}_{1}^{ \pm}}$ in the CMSSM with $m_{0}=4 \mathrm{TeV}, A_{0}=0, \tan \beta=40$, and $\mu>0$. In blue (dashed), the cross section with no cut on $E_{\mathrm{T}}^{\text {miss }}$ or $H_{\mathrm{T}}$ and in red (solid), the cross section after applying a cut on $H_{T}^{\text {jets }} \geq 300 \mathrm{GeV}$

reduced. This is shown by the red (solid) line in Fig. 5: a relatively mild cut on the hadronic $H_{\mathrm{T}}$ reduces the cross section by more than one order of magnitude.

Thus we must conclude that, although electroweak production could contribute efficiently to the production of additional $Z$-bosons, these events cannot overcome the experimental cuts and cannot give rise to the observed signal.

With this channel, we have reviewed all relevant production cross sections of different supersymmetric particles that could potentially explain the signal. The next step would be to calculate the average number of $Z$-bosons per parent particle $Y$, which we use in Eq. (1).

\subsection{Decay of supersymmetric particles to $Z$-bosons}

$Z$-bosons are produced through the decay chains of most MSSM particles, although the number of $Z$-bosons obtained per each supersymmetric particle produced depends on the identity of the supersymmetric particle initially produced and on the supersymmetric spectrum below its mass. The main sources of $Z$-bosons are the decays of neutralinos and charginos and also in some squark decays. The couplings of charginos/neutralinos to $Z$ are given by

$$
\begin{gathered}
\mathcal{L}_{Z \chi \chi}=\frac{g_{2}}{\cos \theta_{W}} Z_{\mu}\left[\chi_{i}^{+} \gamma^{\mu}\left(O_{i j}^{\prime L} P_{L}+O_{i j}^{\prime R} P_{R}\right) \chi_{j}^{-}\right. \\
\left.+\bar{\chi}_{i}^{0} \gamma^{\mu}\left(O_{i j}^{\prime \prime L} P_{L}+O_{i j}^{\prime \prime R} P_{R}\right) \chi_{j}^{0}\right] \\
\text { with } O_{i j}^{\prime L}=-V_{i 1} V_{j 1}^{*}-\frac{1}{2} V_{i 2} V_{j 2}^{*}+\delta_{i j} \sin ^{2} \theta_{W} \\
O_{i j}^{\prime R}=-U_{i 1}^{*} U_{j 1}-\frac{1}{2} U_{i 2}^{*} U_{j 2}+\delta_{i j} \sin ^{2} \theta_{W} \\
O_{i j}^{\prime \prime L}=-\frac{1}{2} N_{i 3} N_{j 3}^{*}+\frac{1}{2} N_{i 4} N_{j 4}^{*} \\
O_{i j}^{\prime \prime R}=-O_{i j}^{\prime \prime L} .
\end{gathered}
$$

Therefore, we can obtain $Z$-bosons in the decays of higgsinolike neutralinos and charginos. For instance, we can define a typical mSugra spectrum with gaugino mass unification at the grand unification scale and all the masses fixed by four parameters $\left(m_{0}, m_{1 / 2}, A_{0}, \tan \beta\right)$ plus the sign of the $\mu$ parameter. In this spectrum, the gaugino masses at the electroweak scale are approximately given by $M_{1} \simeq 0.4 m_{1 / 2}$, $M_{2} \simeq 0.8 m_{1 / 2}, M_{3} \simeq 2.6 m_{1 / 2}$, and the sfermion masses are $m_{\tilde{q}}^{2} \simeq m_{\tilde{u}}^{2} \simeq m_{\tilde{d}}^{2} \simeq m_{0}^{2}+6 m_{1 / 2}^{2}, m_{\tilde{l}}^{2} \simeq m_{0}^{2}+0.5 m_{1 / 2}^{2}$ and $m_{\tilde{e}}^{2} \simeq m_{0}^{2}+0.15 m_{1 / 2}^{2}$ with relatively small dependence on $A_{0}$ and $\tan \beta$. The value of $|\mu|^{2}$ has a stronger dependence on $A_{0}$ and, specially, on $\tan \beta$, for $\tan \beta \sim 5$, we would have $|\mu|^{2} \simeq 0.2 m_{0}^{2}+2.9 m_{1 / 2}^{2}-0.4 A_{0} m_{1 / 2}+0.1 A_{0}^{2}-M_{Z}^{2} / 2$, while for $\tan \beta \sim 40$, we have $|\mu|^{2} \simeq 0.14 m_{0}^{2}+2.6 m_{1 / 2}^{2}-$ $0.4 A_{0} m_{1 / 2}+0.1 A_{0}^{2}-M_{Z}^{2} / 2$. Then, in such a spectrum, the second neutralino will only produce $Z$-bosons through its (relatively small) higgsino component while the two heavier neutralinos can be expected to produce a sizable number of $Z$-bosons. On the other hand, charginos can produce $Z$ bosons both through the wino and from the higgsino component but only in decays of the heavier charginos, as the lightest one will only decay to a $W$-boson and a neutralino (or lepton-slepton if $m_{\tilde{l}} \leq m_{\chi^{+}}$).

In Fig. 6 we can see the values of $\mathcal{N}\left(\chi_{2,3}^{0} \rightarrow Z\right)$ as a function of $m_{\chi_{2,3}^{0}}$ in an mSugra model. Here, we obtain $\mathcal{N}\left(\chi_{2} \rightarrow Z\right)$ around 0.1 , as expected if the higgsino content is relatively small. Then $\mathcal{N}\left(\chi_{3} \rightarrow Z\right)$ can reach at most 0.45 while the other $50 \%$ of the decays go to $\chi^{ \pm} W^{\mp}$. Similarly the heavy charginos produce $Z$-bosons in their decay as can be seen in Fig. 7. In this case $\mathcal{N}\left(\chi_{2}^{+} \rightarrow Z\right)$ can be 0.3 , while we have similar branching ratios to $\chi_{1}^{0} W^{+}$and $\chi_{1}^{+} h$.

Besides chargino and neutralino decays, $Z$-bosons couple also to sfermions through,

$$
\begin{aligned}
\mathcal{L}_{Z \tilde{q}}= & \frac{-i g_{2}}{\cos \theta_{W}}\left(T_{3 I}-e_{I} \sin ^{2} \theta_{W}\right) Z_{\mu} \tilde{q}_{\alpha}^{*} \overleftrightarrow{\partial} \mu \tilde{q}_{\beta} \\
& \times\left(\left(\Gamma_{q L}^{\mathrm{SCKM}}\right)_{I \alpha}^{*}\left(\Gamma_{q L}^{\mathrm{SCKM}}\right)_{I \beta}\right. \\
& \left.+\left(\Gamma_{q R}^{\mathrm{SCKM}}\right)_{I \alpha}^{*}\left(\Gamma_{q R}^{\mathrm{SCKM}}\right)_{I \beta}\right) .
\end{aligned}
$$

As we can see, the $Z$ couplings are chirality diagonal and therefore, in decays, they can only enter through chirality mixing. Although these couplings could be flavor changing, flavor mixing is bounded to be small due to the stringent flavor changing neutral current constraints. Therefore we can expect a sizable amount of $Z$-bosons produced only through chirality mixing in third generation sfermions in decays like $\tilde{t}_{2} \rightarrow \tilde{t}_{1}+Z$, or $\tilde{b}_{2} \rightarrow \tilde{b}_{1}+Z$ in the large $\tan \beta$ regime.

In addition, we obtain $Z$-bosons in the decay chains of strongly produced sparticles. We can obtain $Z$-bosons at different steps of the decay chain, either through the couplings 

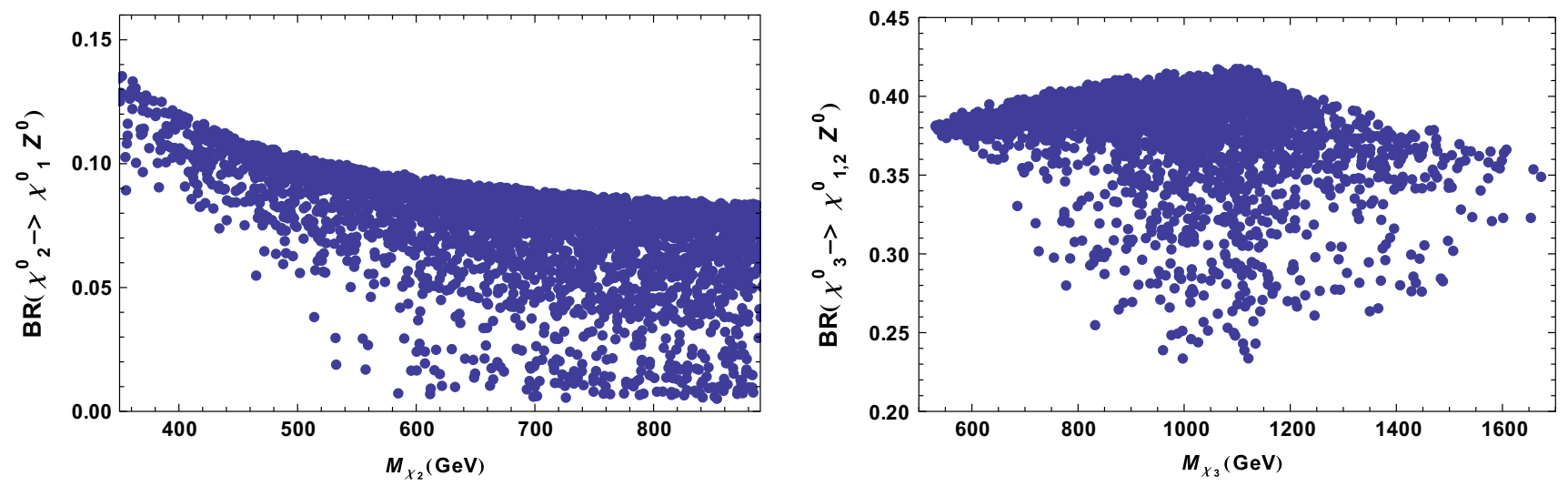

Fig. 6 Average number of $Z$-bosons, $\mathcal{N}\left(Y \rightarrow Z\right.$ ), in the decays of $\chi_{2}^{0}$ (left) and $\chi_{3}^{0}$ (right) for a typical mSugra spectrum as defined in the text

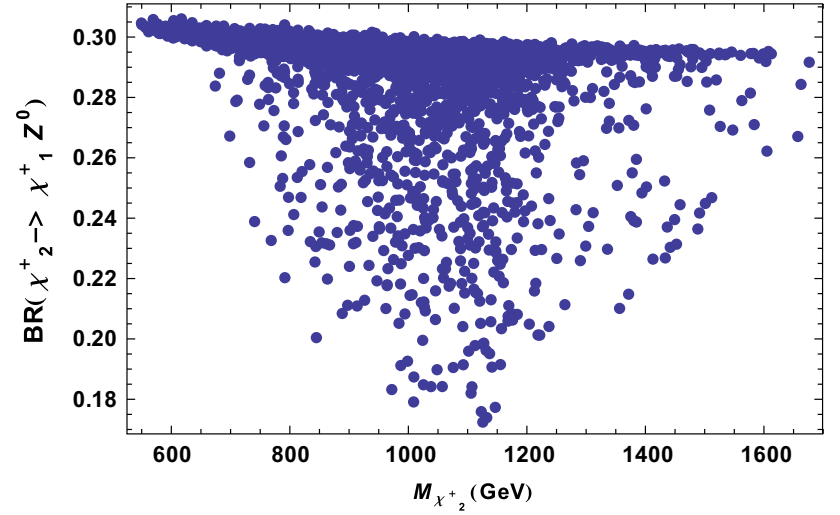

Fig. 7 Average number of $Z$-bosons, $\mathcal{N}\left(\chi_{2}^{+} \rightarrow Z\right)$, in the decays of $\chi_{2}^{+}$for a typical mSugra spectrum as defined in the text

of $Z$ to sfermions or to charginos/neutralinos that we saw above. For instance, if we produce a pair of gluinos a possible decay chain would be $\tilde{g} \rightarrow \tilde{t}_{2} t \rightarrow \tilde{t}_{1} Z t \rightarrow \chi_{2}^{+} b Z t \rightarrow$ $\chi_{1}^{+} Z b Z t \rightarrow \chi_{1}^{0} W^{+} Z b Z t$. Therefore taking into account the corresponding branching ratios, this decay chain would contribute with $2 \times \mathrm{BR}\left(\tilde{g} \rightarrow \chi_{1}^{0} W^{+} Z b Z t\right)$ to $\mathcal{N}(\tilde{g} \rightarrow Z)$, the number of $Z$-bosons produced per $\tilde{g}$ produced.

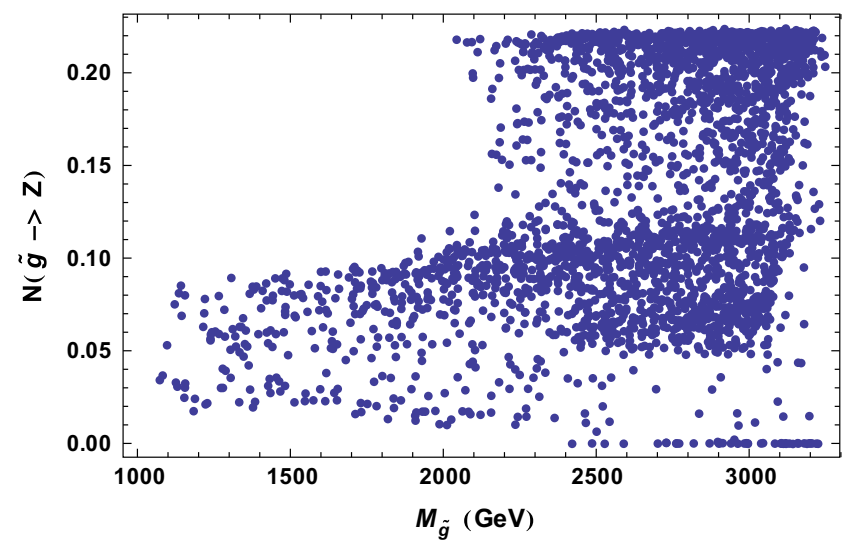

As we can see in Fig. 8, in a typical MSSM spectrum we obtain at most $0.2 \mathrm{Z}$-bosons per stop or gluino while other squarks produce far fewer $Z$-bosons per squark. Although Figs. 6, 7, 8 have been obtained from a mSugra spectrum, the expected number of $Z$-bosons would be very similar in other MSSM versions, as it depends only on the spectrum below the mass of the originally produced $Y$ particle and the content of charginos-neutralinos.

As shown in the previous section, the only possibility to explain the signal is to produce a stop or a gluino pair as the lightest colored sparticle, being all other squarks much heavier and only neutralinos, charginos, and possibly some sleptons can be below the gluino or stop mass. Moreover, given the size of production cross sections consistent with the present searches, we need to obtain nearly one $Z$-boson per $Y$ particle produced. Therefore, from the expected numbers of $Z$-bosons that we have seen in this section, we have to conclude that it is not possible to reproduce the observed signal in a MSSM with a stable (and light) neutralino.

Nevertheless, we can still consider different variations of the MSSM:

Fig. 8 Average number of $Z$-bosons, $\mathcal{N}(Y \rightarrow Z$ ), in gluino (left) and stop (right) decays for a typical mSugra spectrum as defined in the text 
- A first possibility would be to have a light gluino below $1 \mathrm{TeV}$ that can evade the bounds from jets plus missing $E_{T}$ if it decays to a sufficiently heavy neutralino LSP in a sort of compressed spectrum. Under these conditions the gluino would be abundantly produced at LHC and even a small number of $Z$-bosons per gluino could fulfil the requirements to explain the observed signal. However, this would require strongly non-universal gaugino masses and very heavy LSP's and we will not follow this path here.

- A second option is to consider an MSSM where the lightest neutralino decays to a lighter gravitino plus some $Z$ boson. This is the case in gauge mediated MSSM [2730] and it could also be possible in gravity mediated MSSM if the gravitino is lighter than the neutralino which then becomes the NLSP [31]. In this case, the neutralino decays to $Z$-boson and gravitino if it is allowed by phase space and the branching ratio will depend on the lightest neutralino composition. This is the possibility we will explore in the following.

Thus, we will analyze a situation where the neutralino is the next to lightest supersymmetric particle and the LSP is the gravitino. All supersymmetric particles will decay to the lightest neutralino which then decays to gravitino plus a photon, a $Z$-boson or a Higgs. The decay width of the lightest neutralino to photon, $h$ or $Z$ plus gravitino [31-33] is given by

$$
\begin{aligned}
\Gamma\left(\chi_{1}^{0} \rightarrow \gamma \tilde{G}\right)= & \frac{\left|N_{11} \cos \theta_{W}+N_{12} \sin \theta_{W}\right|^{2}}{48 \pi M_{P l}^{2}} \\
& \times \frac{m_{\chi}^{5}}{m_{\tilde{G}}^{2}}\left[1-\frac{m_{\tilde{G}}^{2}}{m_{\chi}^{2}}\right]\left[1+3 \frac{m_{\tilde{G}}^{2}}{m_{\chi}^{2}}\right], \\
\Gamma\left(\chi_{1}^{0} \rightarrow Z \tilde{G}\right)= & \frac{\left|-N_{11} \sin \theta_{W}+N_{12} \cos \theta_{W}\right|^{2}}{48 \pi M_{P l}^{2}} \\
& \times \frac{m_{\chi}^{5}}{m_{\tilde{G}}^{2}} F\left(m_{\chi}, m_{\tilde{G}}, m_{Z}\right), \\
\Gamma\left(\chi_{1}^{0} \rightarrow h \tilde{G}\right)= & \frac{\left|-N_{13} \sin \alpha+N_{14} \cos \alpha\right|^{2}}{96 \pi M_{P l}^{2}} \\
& \times \frac{m_{\chi}^{5}}{m_{\tilde{G}}^{2}} F\left(m_{\chi}, m_{\tilde{G}}, m_{h}\right),
\end{aligned}
$$

with $F(x, y, z)$ a function of the particle masses, irrelevant for our discussion, that can be obtained from [31]. As we can see, if the lightest neutralino is bino-like, $N_{11} \simeq$ 1 , and the mass difference between neutralino and gravitino is larger that the $Z$-mass, the branching ratios are $\mathrm{BR}\left(\chi_{1} \rightarrow \tilde{G} \gamma\right) \simeq \cos ^{2} \theta_{W} \simeq 0.8$ and $\mathrm{BR}\left(\chi_{1} \rightarrow \tilde{G} \mathrm{Z}\right) \simeq$ $\sin ^{2} \theta_{W} \simeq 0.2$. Similarly if the lightest neutralino is wino- like, the branching ratios get exchanged. From this equation we can also see that it is possible to get a very large BR to $Z$-bosons as needed to reproduce the observed signal if $\left(-N_{11} \sin \theta_{W}+N_{12} \cos \theta_{W}\right) \simeq 1$, but this is only possible if the lightest neutralino has a very large wino component.

Although in gauge mediation models the LSP is always the gravitino, the gaugino masses in minimal models are proportional to the gauge couplings and therefore the LSP is mostly bino with small wino and higgsino components. Then we will have to consider other extensions of the gauge mediation idea, like the so-called general gauge mediation (GGM) where the gaugino and sfermion masses depend on hidden sector current correlators which can be different for different gauge groups or particle representations. As we will show in the following section, in these models it is possible to obtain general neutralino NLSP as required to explain the excess.

\section{A possible explanation in general gauge mediation}

Minimal gauge mediation predicts that all scalar and gaugino masses originate from a single scale and powers of the gauge couplings [30]. Recently a model-independent generalization of gauge mediation was proposed under the name of general gauge mediation $[34,35]$, where all the dependence of soft masses on the hidden sector is encoded in three real and three complex parameters obtained from a small set of currentcurrent correlators. In these models the gaugino and sfermion masses are given by

$$
\begin{aligned}
& M_{r}=g_{r}^{2} M_{s} \tilde{B}_{r}^{1 / 2}(0) \\
& m_{\tilde{f}}^{2}=g_{1}^{2} Y_{f} \zeta+\sum_{r=1}^{3} g_{r}^{2} \mathcal{C}_{2}(f \mid r) M_{s}^{2} \tilde{A}_{r},
\end{aligned}
$$

with

$\tilde{A}_{r}=-\frac{1}{16 \pi^{2}} \int d y\left(3 \tilde{C}_{1}^{(r)}(y)-4 \tilde{C}_{1 / 2}^{(r)}(y)+\tilde{C}_{0}^{(r)}(y)\right)$,

$\tilde{B}_{r}^{1 / 2}(0), C_{\rho}^{(r)}(y)$ (with $\rho=0,1 / 2,1$, corresponding to scalar, fermion and vector) are associated with the currentcurrent correlators in the hidden sector, $\zeta$ is a possible FayetIlliopoulos term ( $\zeta=0$ in the following), $\mathcal{C}_{2}(f \mid r)$ the quadratic Casimirs and $M_{S}$ a characteristic SUSY-breaking scale in the hidden sector.

Having six parameters, $\tilde{B}_{r}^{1 / 2}(0)$ and $\tilde{A}_{r}$, to fix the soft masses in the observable sector, it is clear now that we have much more freedom in GGM [36-39] and, in particular, we have

$$
\frac{M_{1}}{g_{1}^{2}} \neq \frac{M_{2}}{g_{2}^{2}} \neq \frac{M_{3}}{g_{3}^{2}}
$$


Table 1 SUSY spectrum in the GGM1 parameter point

\begin{tabular}{lllllllll}
\hline Particle & $\tilde{g}$ & $\chi_{1}^{0}$ & $\chi_{2}^{0}$ & $\chi_{3}^{0}$ & $\chi_{4}^{0}$ & $\chi_{1}^{ \pm}$ & $\chi_{2}^{ \pm}$ & $\tilde{G}^{ \pm}$ \\
\hline Mass $(\mathrm{GeV})$ & 1088.0 & 428.4 & 431.34 & 1357.0 & 1360.9 & 429.1 & 1361.0 & $4.8 \times 10^{-9}$ \\
\hline Particle & $\tilde{q}_{L}$ & $\tilde{q}_{R}$ & $\tilde{b}_{1}$ & $\tilde{b}_{2}$ & $\tilde{t}_{1}$ & $\tilde{t}_{2}$ & $\tilde{l}_{L}$ & \\
\hline Mass $(\mathrm{GeV})$ & 3006 & 2957 & 2876 & 2952 & 2716 & 2881 & 5863 & 5328 \\
\hline Particle & $h$ & $H$ & $A$ & $H^{+}$ & & & \\
\hline Mass $(\mathrm{GeV})$ & 119.4 & 1471 & 1471 & 1473 & & & \\
\hline
\end{tabular}

as required to reproduce the observed signal at ATLAS. In particular, we need the NLSP to decay to gravitino and a $Z$ boson with a branching ratio close to one. Fortunately, this is possible in GGM as shown in Refs. [39,40].

In this GGM scenario we have used SPheno-3.3.3 [41,42] to obtain the full supersymmetric spectrum at LHC energies. We define a first parameter point GGM1 with the following parameters: $M_{s}=400 \mathrm{TeV}, \tilde{B}_{1}^{1 / 2}=\tilde{A}_{1}=309 \mathrm{TeV}, \tilde{B}_{2}^{1 / 2}=$ $\tilde{A}_{2}=151 \mathrm{TeV}, \tilde{B}_{3}^{1 / 2}=129 \mathrm{TeV}, \tilde{A}_{3}=316 \mathrm{TeV}$ and $\tan \beta=$ 9.8. With these parameters we obtain the spectrum shown in Table 1. With respect to this spectrum, some comments are in order:

1. The two lightest neutralinos and the lightest chargino are very similar in mass, $\sim 430 \mathrm{GeV}$ and this allows a large neutrino mixing as required. In fact the neutralino mixing matrix is given by

$$
N_{i j} \simeq\left(\begin{array}{cccc}
-0.51 & 0.85 & -0.076 & 0.031 \\
0.86 & 0.51 & -0.0024 & 0.0071 \\
-0.015 & 0.028 & 0.71 & 0.71 \\
-0.037 & 0.065 & 0.70 & -0.71
\end{array}\right)
$$

On the other hand the relatively large NLSP mass is needed to overcome the $E_{\mathrm{T}}^{\text {miss }}$ cut.

2. The gluino is relatively light $m_{\tilde{g}}=1088.0 \mathrm{GeV}$ which allows for a sizable production cross section and taking into account the squark masses of order $\sim 3 \mathrm{TeV}$. As we can see in Ref. [15], this mass would be allowed at $1 \sigma$ in a simplified MSSM with gluino-squark-neutralino.

3. The lightest Higgs mass must reproduce the observed value at $\mathrm{LHC}$ of $m_{h} \simeq 125 \mathrm{GeV}$ and in this spectrum it reaches only $119.4 \mathrm{GeV}$. This problem (typical in minimal gauge mediation models) can be solved either by increasing the stop masses taking a larger $\tilde{A}_{3}$ or assuming extra operators in the Higgs sector, as the dimension-5 operators proposed by Dine et al. [43]. Here, we assume that this problem is solved by one of these mechanisms, given it does not affect the observed phenomenology on the $Z$-peak.
Under these conditions, the lightest neutralino width is $\Gamma_{\chi_{1}}=4.097 \times 10^{-10} \mathrm{GeV}$ and the decay branching ratios are $\operatorname{BR}\left(\chi_{1}^{0} \rightarrow \tilde{G} \gamma\right)=1.14 \times 10^{-3}, \operatorname{BR}\left(\chi_{1}^{0} \rightarrow \tilde{G} Z\right)=0.997$, and $\operatorname{BR}\left(\chi_{1}^{0} \rightarrow \tilde{G} h\right)=1.35 \times 10^{-3}$. Therefore, gluinos are produced at LHC with a cross section of $8.4 \pm 1.6 \mathrm{fb}$ at NLL+NLO and after going through different decay chains all of them produce a $Z$-boson plus a gravitino. In this case, the strong production represents approximately $20 \%$ of the total production cross section.

We simulate the production of supersymmetric particles at LHC at $8 \mathrm{TeV}$ (LHC8) with this spectrum using Pythia 8.1 [44] with Prospino2 [16,17,45,46] K-factors and the response of the ATLAS detector using Delphes [12]. The selection of events for this study is done as close as possible to that performed in ATLAS [9]. The dashed red line in Figs. 9 and 10 shows the $H_{\mathrm{T}}$ and $E_{\mathrm{T}}^{\text {miss }}$ distributions respectively for the GGM1 point after applying all the selection detailed in Sect. 2 except for the $H_{\mathrm{T}}$ and $E_{\mathrm{T}}^{\text {miss }}$ cuts. In the $H_{\mathrm{T}}$ distribution, we can distinguish the two peaks corresponding to electroweak production at low $H_{\mathrm{T}}$ values and gluino production at higher $H_{\mathrm{T}}$. From here, we can expect that the cut on $H_{\mathrm{T}}$ will eliminate most of the electroweak production but not the gluino production. This can be seen in Fig. 10 , where the $E_{\mathrm{T}}^{\text {miss }}$ distribution is presented separately for strongly produced events (solid blue line) and for the electroweak component of the production (dashed red line) for the same selection as in Fig. 9 but after applying the $H_{\mathrm{T}}$ cut, i.e. the final selection except for the cut on $E_{\mathrm{T}}^{\text {miss }}$. The electroweak component is significantly reduced by the $H_{\mathrm{T}}$ cut while mainly only events coming from strong production survive the cut, as expected. In fact, in this simulation of point GGM1, $99 \%$ of the gluino points and only $11 \%$ of the electroweak points have survived the $H_{\mathrm{T}}$ cut. We see that the peak in the $E_{\mathrm{T}}^{\text {miss }}$ distribution is approximately at $m_{\chi_{1}^{0}} / 2$, and, for $m_{\chi_{1}^{0}}=425 \mathrm{GeV}$, a reasonable fraction of the events will survive the $E_{\mathrm{T}}^{\text {miss }}$ cut at $225 \mathrm{GeV}$. In the simulation, $65 \%$ of the gluino point and $53 \%$ of the electroweak points survive this cut. However, due the relatively small production cross section, the final number of events is small. In this simulation and after applying all relevant experimental cuts, an 

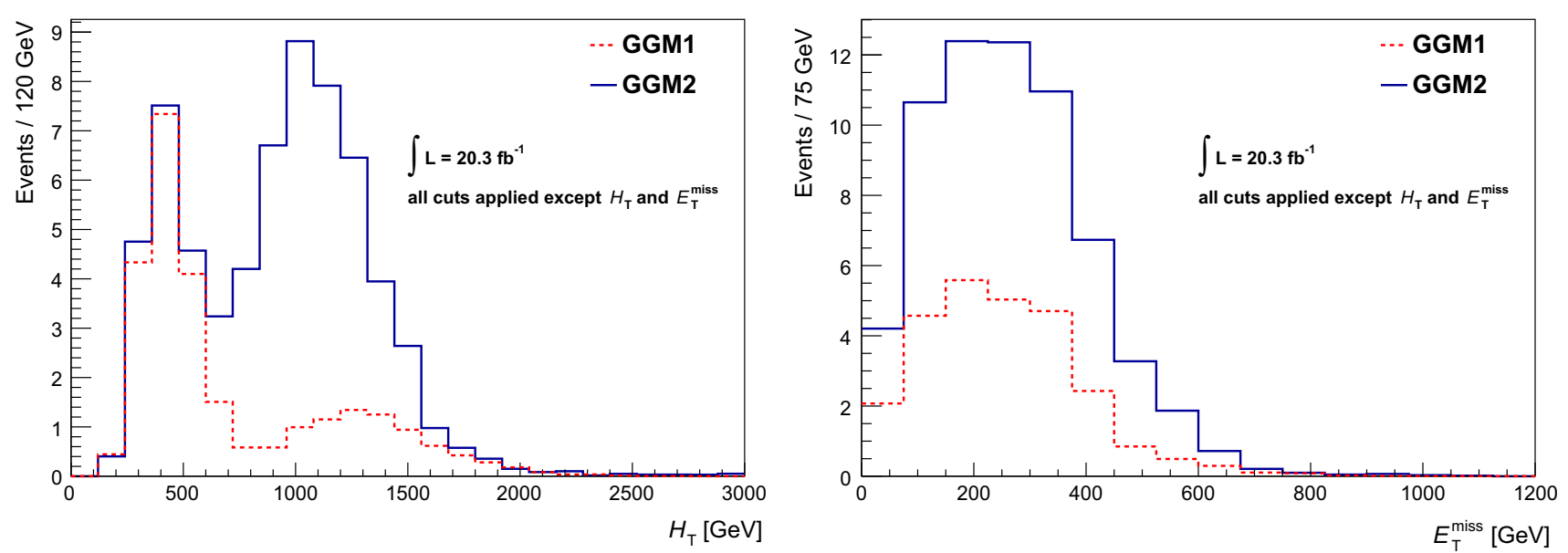

Fig. $9 H_{\mathrm{T}}($ left $)$ and $E_{\mathrm{T}}^{\text {miss }}($ right $)$ distributions after applying the selection detailed in Sect. 2, except for the cuts on $H_{\mathrm{T}}$ and $E_{\mathrm{T}}^{\mathrm{miss}}$. The GGM1 point is represented by the dashed red line and the GGM2 point, by the solid blue line

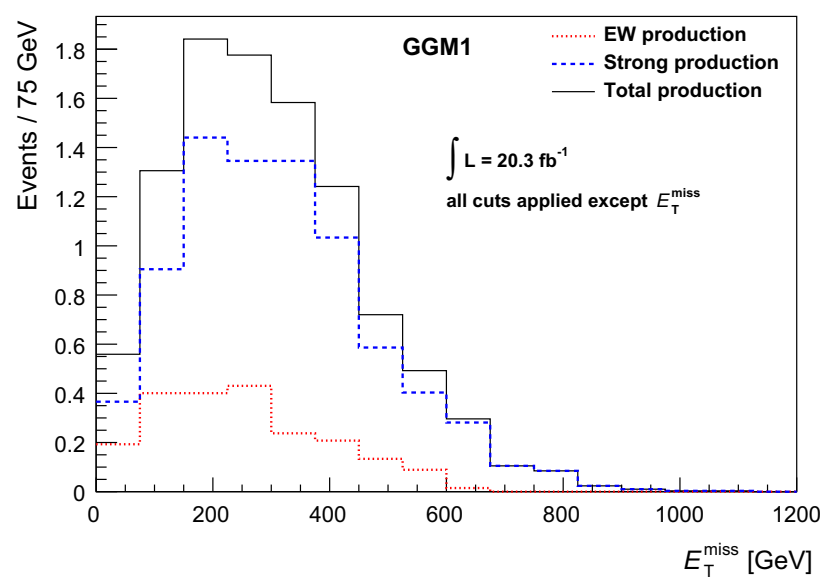

Fig. $10 E_{\mathrm{T}}^{\mathrm{miss}}$ distribution corresponding to the GGM1 point from strong production (solid blue line) and electroweak production (dashed red line) with the same selection as in Fig. 9, after applying the $H_{\mathrm{T}}>600 \mathrm{GeV}$ cut

expected signal of $6.34 \pm 1.02$ lepton pairs is found, to be compared with the observed excess of $19.4 \pm 3.2$. This number of surviving events was obtained at NLO with Pythia and Prospino 2 but, unfortunately, it is still too low to explain the observations.

Trying to obtain a model able to account for the excess, we consider a second point in our GGM scenario with a lighter gluino. The GGM2 point is obtained with $M_{s}=400 \mathrm{TeV}$, $\tilde{B}_{1}^{1 / 2}=\tilde{A}_{1}=309 \mathrm{TeV}, \tilde{B}_{2}^{1 / 2}=\tilde{A}_{2}=150 \mathrm{TeV}, \tilde{B}_{3}^{1 / 2}=$ $110 \mathrm{TeV}, \tilde{A}_{3}=270 \mathrm{TeV}$ and $\tan \beta=9.8$. With these parameters we obtain the spectrum shown in Table 2.

The neutralino mixing matrix in point GGM2 is similar to the corresponding mixing matrix in GGM1, and the $\operatorname{BR}\left(\chi_{1}^{0} \rightarrow \tilde{G} Z\right)=0.94$. However, gluino is now much lighter and the gluino-gluino cross section is now (41.6 \pm 7.5$)$ with Prospino at NLL+NLO, thus we can expect many more

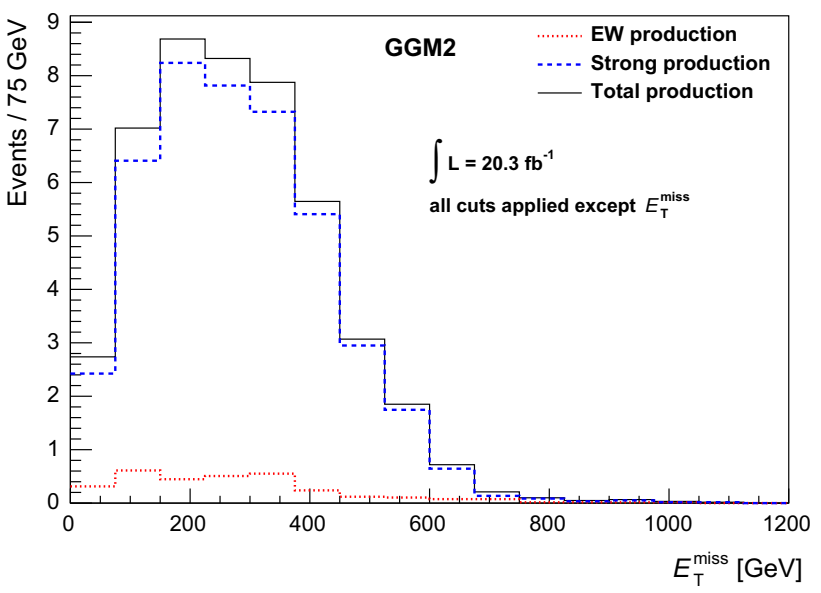

Fig. $11 E_{\mathrm{T}}^{\text {miss }}$ distribution corresponding to the GGM2 point from strong production (solid blue line) and electroweak production (dashed red line) with the same selection as in Fig. 9, after applying the $H_{\mathrm{T}}>600 \mathrm{GeV}$ cut

gluino pairs to be produced and a larger contribution in the final selection for this point.

The simulation for this GGM2 point is presented by the solid blue line in Fig. 9. The $H_{\mathrm{T}}$ distribution peaks at slightly lower values than in the case of GGM1, due to the slightly lower gluino mass, but it is still enough to overcome the $H_{\mathrm{T}}$ cut at $600 \mathrm{GeV}$. The $E_{\mathrm{T}}^{\text {miss }}$ distributions are similar for both GGM points, due to the very similar neutralino masses in both cases. However, in the case of GGM2 the strong production cross section is larger and much more important in relation with the electroweak production: for the GGM2 point, the strong production represents $\sim 55 \%$ of the total cross section. As seen in Fig. 11, after applying the selection we obtain an expected signal of $28.0 \pm 4.7$ events, slightly over the excess reported by ATLAS, showing that a signal point defined along these characteristics can be able to reproduce the excess. 
Table 2 SUSY spectrum for the point GGM2

\begin{tabular}{lllllllll}
\hline Particle & $\tilde{g}$ & $\chi_{1}^{0}$ & $\chi_{2}^{0}$ & $\chi_{3}^{0}$ & $\chi_{4}^{0}$ & $\chi_{1}^{ \pm}$ & $\chi_{2}^{ \pm}$ & $\tilde{G}^{ \pm}$ \\
\hline Mass $(\mathrm{GeV})$ & 911.4 & 424.9 & 432.7 & 1111.8 & 1117.1 & 425.8 & 1117.2 & $4.8 \times 10^{-9}$ \\
\hline Particle & $\tilde{q}_{L}$ & $\tilde{q}_{R}$ & $\tilde{b}_{1}$ & $\tilde{b}_{2}$ & $\tilde{t}_{1}$ & $\tilde{t}_{2}$ & $\tilde{l}_{L}$ & \\
\hline Mass $(\mathrm{GeV})$ & 2510 & 2470 & 2400 & 2450 & 2250 & 2400 & 5890 & 5360 \\
\hline Particle & $h$ & $H$ & $A$ & $H^{+}$ & & & \\
\hline Mass $(\mathrm{GeV})$ & 118.1 & 1250 & 1250 & 1253 & & & \\
\hline
\end{tabular}

We have to emphasize here that it is not difficult to obtain the observed excess for light gluino masses, and a gluino mass in between the two presented examples, $m_{\tilde{g}} \in[900,1100]$ $\mathrm{GeV}$, could reproduce the observed signal. However, these points may be in conflict with direct searches of jets plus $E_{\mathrm{T}}^{\text {miss }}$ [13-15]. There is a tension between this excess and the bounds from gluino searches in jets plus $E_{\mathrm{T}}^{\text {miss }}$. To quantify more accurately this tension, we use the program Checkmate [47] that allows one to compare the different points of the model with various experimental analyses $[47,48]$ determining whether the point is excluded or not at $95 \%$ C.L.. We applied the constraints on jets plus $E_{\mathrm{T}}^{\text {miss }}$ from ATLAS studies: ATLAS.1308.1841 [49,50], ATLAS.1308.2631 [51], ATLAS.1407.0583 [24] and ATLAS.1405.7875 [15]. In fact, we find that this point is indeed excluded by the analysis ATLAS.1405.7875, in the signal region with six (or more) jets, with an $r \equiv \frac{S-1.96 \Delta S}{S_{0 b s}^{95}}=2.0$, where $S$ is the total number of expected signal events, $\Delta S$ is the total $1 \sigma$ uncertainty on this number and $S_{o b s}^{95}$ is the experimentally measured $95 \%$ confidence limit on signal events.

Thus, we have to find another parameter point able to provide the signal, but satisfying all the present constraints on jets plus $E_{\mathrm{T}}^{\text {miss }}$. The main difficulty in the GGM scenario is that each gluino decay produces typically four or more jets with large $p_{\mathrm{T}}$ and $E_{\mathrm{T}}^{\text {miss }}$. Therefore, these points clash with constraints from observables with a large number of jets (plus $E_{\mathrm{T}}^{\text {miss }}$ ), which have low backgrounds in the Standard Model. On the contrary, constraints with fewer jets are easier to satisfy because of larger backgrounds. Then our strategy will be to compress sufficiently the spectrum to reduce the number of (observable) jets. This can be done by increasing the lightest neutralino mass closer to the gluino mass, so that the jets in the decays $\tilde{g} \rightarrow \chi_{1}+$ jets have smaller $p_{\mathrm{T}}$. With this goal, we construct a third GGM point. The GGM3 point is obtained with $M_{s}=4160 \mathrm{TeV}, \tilde{B}_{1}^{1 / 2}=\tilde{A}_{1}=662.5 \mathrm{TeV}$, $\tilde{B}_{2}^{1 / 2}=\tilde{A}_{2}=344 \mathrm{TeV}, \tilde{B}_{3}^{1 / 2}=117 \mathrm{TeV}, \tilde{A}_{3}=330 \mathrm{TeV}$ and $\tan \beta=34$.4. With these parameters we obtain the spectrum shown in Table 3.

For this point, the $\operatorname{BR}\left(\chi_{1}^{0} \rightarrow \tilde{G} Z\right)=0.98$ and the gluino production cross section is $(22.8 \pm 3.3)$ fb with Prospino at

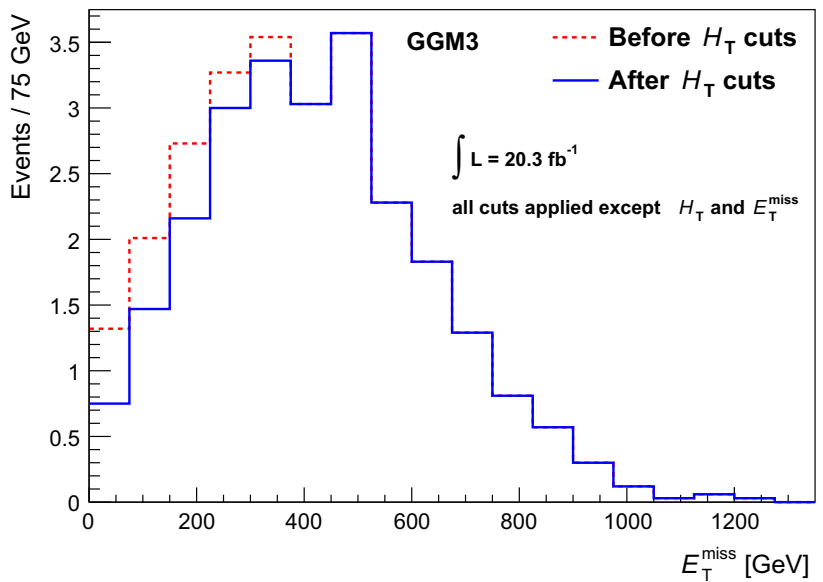

Fig. $12 E_{\mathrm{T}}^{\text {miss }}$ distribution corresponding to the GGM3 point from strong production with the same selection as in Fig. 9, before (red dashed) and after (solid blue) applying the $H_{\mathrm{T}}>600 \mathrm{GeV}$ cut. Notice that electroweak production is negligible for the neutralino masses in GGM3

NLL+NLO. In this case, the signal is lower than in GGM2 but we still get $(13.1 \pm 2.2)$ events, as shown in Fig. 12, well above the experimentally measured $95 \%$ confidence limit on signal events.

Concerning the experimental searches on jets plus MET, according to Checkmate this point is allowed with an $r=1.0$ with the best signal region being two jets plus $E_{\mathrm{T}}^{\text {miss }}$ from ATLAS.1405.7875. Moreover, in the equivalent search by CMS of two leptons, jets, and $E_{\mathrm{T}}^{\text {miss }}$, CMS-SUS-14-014 [11], we obtain $r=1.2$ in Checkmate. This result shows some tension with the ATLAS positive signal from the bin with large $E_{\mathrm{T}}^{\text {miss }}$ in the CMS analysis, but still consistent with observations slightly above $95 \%$ C.L.

Therefore, we have proved that it is indeed possible to construct a supersymmetric model that accommodates the observed excess of lepton pairs on the Z-peak. The simulations presented here are only a proof of existence and the final model may be very different. Nevertheless, this model will have to share the main features of the examples that we presented here. 
Table 3 SUSY spectrum for the point GGM3

\begin{tabular}{lllllllll}
\hline Particle & $\tilde{g}$ & $\chi_{1}^{0}$ & $\chi_{2}^{0}$ & $\chi_{3}^{0}$ & $\chi_{4}^{0}$ & $\chi_{1}^{ \pm}$ & $\chi_{2}^{ \pm}$ & $\tilde{G}^{2}$ \\
\hline Mass $(\mathrm{GeV})$ & 985.4 & 905.7 & 918.7 & 1175.3 & 1190.6 & 910.2 & 1187.9 & $\tilde{l}_{L}$ \\
\hline Particle & $\tilde{q}_{L}$ & $\tilde{q}_{R}$ & $\tilde{b}_{1}$ & $\tilde{b}_{2}$ & $\tilde{t}_{1}$ & $\tilde{t}_{2}$ & $\tilde{l}_{R}$ & 1330 \\
\hline Mass $(\mathrm{GeV})$ & 3140 & 2970 & 2820 & 2920 & 2630 & 2920 & 1210 \\
\hline Particle & $h$ & $H$ & $A$ & $H^{+}$ & & & \\
\hline Mass $(\mathrm{GeV})$ & 121.0 & 1489 & 1489 & 1491 & & & \\
\hline
\end{tabular}

\section{Prospects for SUSY at LHC13}

As we have shown in the previous sections, the excess observed in ATLAS, if due to SUSY, requires a gluino of a mass $\sim 1 \mathrm{TeV}$ producing nearly one $Z$-boson per gluino in its decay. This scenario would also require relatively heavy squarks of the first generation with $m_{\tilde{q}} \gtrsim 2.5 \mathrm{TeV}$. If this is indeed the correct explanation to the observed excess, such light gluinos would be abundantly produced at Run II in LHC together with other SUSY particles. Therefore, the results obtained at $p p$ collisions at $13 \mathrm{TeV}$ will immediately confirm or reject this supersymmetric explanation of the ATLAS excess.

Using NLL-Fast with the spectrum of point GGM1, the gluino pair production cross section at $8 \mathrm{TeV}$ was $\sigma(p p \rightarrow$ $\tilde{g} \tilde{g})_{\mathrm{LHC} 8}^{\mathrm{NLL}+\mathrm{NLO}}=7.6 \pm 1.3 \mathrm{fb}$ at NLL+NLO. Similarly the production cross section at $13 \mathrm{TeV}$ would be $\sigma(p p \rightarrow$ $\tilde{g} \tilde{g})_{\mathrm{LHC13}}^{\mathrm{NLL}+\mathrm{NLO}}=150 \pm 16 \mathrm{fb}$, that is, we would expect to produce 20 times more gluinos at LHC13 for the same integrated luminosity. Repeating the same exercise with point GGM3, we have a gluino pair production cross section at $8 \mathrm{TeV}$ of $\sigma(p p \rightarrow \tilde{g} \tilde{g})_{\mathrm{LHC} 8}^{\mathrm{NLL}+\mathrm{NLO}}=22.8 \pm 3.3 \mathrm{fb}$ while the production cross section at $13 \mathrm{TeV}$ would be $\sigma(p p \rightarrow$ $\tilde{g} \tilde{g})_{\mathrm{LHC} 13}^{\mathrm{NLL}+\mathrm{NLO}}=344 \pm 44 \mathrm{fb}$. In this point, the cross section increases by a factor $\sim 15$ in going from 8 to $13 \mathrm{TeV}$ center of mass energy. In any case, for both points this would have unambiguous signatures, both on the $Z$-peak with a scaling of the signal found at LHC8 and in direct searches for gluinos using jets plus missing $E_{T}$ in the extension of the analysis in [13-15].

To confirm that it is indeed SUSY behind the excess found at the $Z$-peak, we should search for other sparticles at LHC13. As shown in Sect. 3.1, squarks of the first generation are preferred be heavy to increase the gluino production cross section, namely above $2.5 \mathrm{TeV}$. Then their production cross section at LHC13 would be around $1 \mathrm{fb}$ which would make direct detection very challenging. Charginos and neutralinos are expected to be below the gluino mass and in some cases could be abundantly produced at LHC. Although our analysis does not fix the masses of $\chi_{1}^{ \pm}$and $\chi_{1,2}^{0}$, they are expected to be rather heavy $m_{\chi_{1}^{0}} \gtrsim 300 \mathrm{GeV}$ and degenerate. In some cases, the production cross section could be large. For instance $\sigma\left(p p \rightarrow \tilde{\chi}_{1}^{+} \chi_{1}^{0}\right)_{\mathrm{LHC} 8}^{\mathrm{NLO}}=60.2 \pm 0.9 \mathrm{fb}$ and $\sigma\left(p p \rightarrow \tilde{\chi}_{1}^{+} \chi_{1}^{0}\right)_{\mathrm{LHC13}}^{\mathrm{NLO}}=154 \pm 5 \mathrm{fb}$ calculated with Prospino for $m_{\chi_{1}^{0}}=313 \mathrm{GeV}$ and $m_{\chi_{1}^{+}}=314 \mathrm{GeV}$ (with large $\tilde{W}$ component in $\chi_{1}^{0}$ ). Notice that, as explained above, electroweak production is also large for these points at $8 \mathrm{TeV}$, but it is eliminated by the cuts on $H_{\mathrm{T}}$ and number of jets. Thus, a large electroweak production could be expected at LHC13 and dedicated searches should be encouraged, specially taking into account the requirement of a large production of $Z$-bosons in chargino and neutralino decays. Finally, the signal does not constrain the masses of sleptons or third generation squarks and, a priory, we cannot make any prediction on their production at $\mathrm{LHC13}$.

Before closing, we should comment on the nature of dark matter in our scenario. As we have seen the signal seems to prefer a non-stable neutralino decaying to a very light gravitino and a $Z$-boson. Under these conditions the neutralino mass has no relation with the dark matter abundance of the universe and its role as dark matter component would be played by the gravitino. The gravitino mass is not bounded by the observed signal but regardless of its exact mass, unfortunately no signal of dark matter is to be expected in direct search experiments.

\section{Conclusions}

Recently the ATLAS experiment announced a $3 \sigma$ excess at the $Z$-peak consistent of 29 pairs of leptons observed to be compared with $10.6 \pm 3.2$ expected lepton pairs. No excess outside the $Z$-peak was observed. By trying to explain this signal with SUSY we found that only relatively light gluinos, $m_{\tilde{g}} \lesssim 1.2 \mathrm{TeV}$, together with a heavy neutralino NLSP of $m_{\tilde{\chi}} \gtrsim 400 \mathrm{GeV}$ decaying predominantly to $Z$-boson plus a light gravitino, such that nearly every gluino produces at least one $Z$-boson in its decay chain, could do it.

Unfortunately, this is not possible withing minimal SUSY models, as mSugra, minimal gauge mediation or anomaly 
mediation. The requirement of a neutralino NLSP decaying to $Z$ plus gravitino points to models of general gauge mediation as the simplest possibility. We have shown that a model of this class is able to reproduce the observed signal overcoming all the experimental cuts. Needless to say, more sophisticated models could also reproduce the signal, however, they will always share the above mentioned features, i.e. light gluinos (or heavy particles with a strong production cross section) with an effective $\mathcal{N}(\tilde{g} \rightarrow Z) \simeq 1$.

Acknowledgments The authors are grateful to Francisco Campanario for useful discussions and Emma Torró for her contributions in an early version of this paper. GB, JB and OV acknowledge support from the Ministry of Economy and Competitiveness (MINECO) and FEDER (EC) Grants FPA2011-23596 and FPA2014-54459-P. GB, JB, VAM and OV from the Generalitat Valenciana under Grant PROMETEOII/2013/017. GB acknowledges partial support from the European Union FP7 ITN INVISIBLES (Marie Curie Actions, PITN- GA-2011289442). ER and VAM acknowledge support by MINECO under the project FPA2012-39055-C02-01. VAM acknowledges support by the Spanish National Research Council (CSIC) under the JAE-Doc program co-funded by the European Social Fund (ESF).

Open Access This article is distributed under the terms of the Creative Commons Attribution 4.0 International License (http://creativecomm ons.org/licenses/by/4.0/), which permits unrestricted use, distribution, and reproduction in any medium, provided you give appropriate credit to the original author(s) and the source, provide a link to the Creative Commons license, and indicate if changes were made. Funded by SCOAP ${ }^{3}$.

\section{References}

1. W.W. Armstrong et al. [ATLAS collaboration], CERN-LHCC-9443

2. G. Aad et al. [ATLAS Collaboration], JINST 3, S08003 (2008). doi:10.1088/1748-0221/3/08/S08003

3. G. Aad et al. [ATLAS collaboration]. arXiv:0901.0512 [hep-ex]

4. [CMS Collaboration], CERN-LHCC-94-38, CERN-LHCC-P-1

5. CERN, Large Hadron Collider conceptual design report No AC-9505 (1995). http://cds.cern.ch/record/291782/files/cm-p00047618. pdf

6. L. Evans, P. Bryant, JINST 3, S08001 (2008). doi:10.1088/ 1748-0221/3/08/S08001

7. G. Aad et al. [ATLAS Collaboration], Phys. Lett. B 716, 1 (2012). doi:10.1016/j.physletb.2012.08.020. arXiv:1207.7214 [hep-ex]

8. S. Chatrchyan et al. [CMS Collaboration], Phys. Lett. B 716, 30 (2012). doi:10.1016/j.physletb.2012.08.021. arXiv:1207.7235 [hep-ex]

9. G. Aad et al. [ATLAS Collaboration], Eur. Phys. J. C 75(7), 318 (2015). doi:10.1140/epjc/s10052-015-3661-9. arXiv:1503.0329 [hep-ex]

10. G. Aad et al. [ATLAS Collaboration], Eur. Phys. J. C 75(10), 463 (2015). doi:10.1140/epjc/s10052-015-3518-2. arXiv:1503.0329 [hep-ex]

11. V. Khachatryan et al. [CMS Collaboration], JHEP 1504, 124 (2015). doi:10.1007/JHEP04(2015)124. arXiv:1502.0603 [hepex]

12. J. de Favereau et al. [DELPHES 3 Collaboration], JHEP 1402, 057 (2014). doi:10.1007/JHEP02(2014)057. arXiv:1307.6346 [hepex]
13. V. Khachatryan et al. [CMS Collaboration], Phys. Rev. D 91, 052018 (2015). doi:10.1103/PhysRevD.91.052018. arXiv: 1502.0030 [hep-ex]

14. G. Aad et al. [ATLAS Collaboration], JHEP 1504, 116 (2015). doi:10.1007/JHEP04(2015)116. arXiv:1501.0355 [hep-ex]

15. G. Aad et al. [ATLAS Collaboration], JHEP 1409, 176 (2014). doi:10.1007/JHEP09(2014)176. arXiv:1405.7875 [hep-ex]

16. W. Beenakker, R. Hopker, M. Spira, P.M. Zerwas, Nucl. Phys. B 492, 51 (1997). doi:10.1016/S0550-3213(97)80027-2. arXiv:hep-ph/9610490

17. W. Beenakker, M. Kramer, T. Plehn, M. Spira, P.M. Zerwas, Nucl. Phys. B 515, 3 (1998). doi:10.1016/S0550-3213(98)00014-5. arXiv:hep-ph/9710451

18. A. Kulesza, L. Motyka, Phys. Rev. Lett. 102, 111802 (2009). doi:10.1103/PhysRevLett.102.111802. arXiv:0807.2405 [hep-ph]

19. A. Kulesza, L. Motyka, Phys. Rev. D 80, 095004 (2009). doi:10. 1103/PhysRevD.80.095004. arXiv:0905.4749 [hep-ph]

20. W. Beenakker, S. Brensing, M. Kramer, A. Kulesza, E. Laenen, I. Niessen, JHEP 0912, 041 (2009). doi:10.1088/1126-6708/2009/ 12/041. arXiv:0909.4418 [hep-ph]

21. W. Beenakker, S. Brensing, M. Kramer, A. Kulesza, E. Laenen, I. Niessen, JHEP 1008, 098 (2010). doi:10.1007/ JHEP08(2010)098. arXiv:1006.4771 [hep-ph]

22. W. Beenakker, S. Brensing, M. n. Kramer, A. Kulesza, E. Laenen, L. Motyka and I. Niessen. Int. J. Mod. Phys. A 26, 2637 (2011). doi:10.1142/S0217751X11053560. arXiv:1105.1110 [hep-ph]

23. V. Khachatryan et al. [CMS Collaboration], Phys. Lett. B 736, 371 (2014). doi:10.1016/j.physletb.2014.07.053. arXiv:1405.3886 [hep-ex]

24. G. Aad et al. [ATLAS Collaboration], JHEP 1411, 118 (2014). doi:10.1007/JHEP11(2014)118. arXiv:1407.0583 [hep-ex]

25. V. Khachatryan et al. [CMS Collaboration], Phys. Rev. D 90(9), 092007 (2014). doi:10.1103/PhysRevD.90.092007. arXiv: 1409.3168 [hep-ex]

26. G. Aad et al. [ATLAS Collaboration], JHEP 1405, 071 (2014). doi:10.1007/JHEP05(2014)071. arXiv:1403.5294 [hep-ex]

27. M. Dine, A. E. Nelson, Phys. Rev. D 48, 1277 (1993). doi:10.1103/ PhysRevD.48.1277. arXiv:hep-ph/9303230

28. M. Dine, A.E. Nelson, Y. Shirman, Phys. Rev. D 51, 1362 (1995). doi:10.1103/PhysRevD.51.1362. arXiv:hep-ph/9408384

29. M. Dine, A.E. Nelson, Y. Nir, Y. Shirman, Phys. Rev. D 53, 2658 (1996). doi:10.1103/PhysRevD.53.2658. arXiv:hep-ph/9507378

30. G.F. Giudice, R. Rattazzi, Phys. Rept. 322, 419 (1999). doi:10. 1016/S0370-1573(99)00042-3. arXiv:hep-ph/9801271

31. J.L. Feng, S. Su, F. Takayama, Phys. Rev. D 70, 075019 (2004). doi:10.1103/PhysRevD.70.075019. arXiv:hep-ph/0404231

32. T. Moroi. arXiv:hep-ph/9503210

33. J.R. Ellis, K.A. Olive, Y. Santoso, V.C. Spanos, Phys. Lett. B 588, 7 (2004). doi:10.1016/j.physletb.2004.03.021. arXiv:hep-ph/0312262

34. P. Meade, N. Seiberg, D. Shih, Prog. Theor. Phys. Suppl. 177, 143 (2009). doi:10.1143/PTPS.177.143. arXiv:0801.3278 [hep-ph]

35. M. Buican, P. Meade, N. Seiberg, D. Shih, JHEP 0903, 016 (2009). doi:10.1088/1126-6708/2009/03/016. arXiv:0812.3668 [hep-ph]

36. L.M. Carpenter. arXiv:0812.2051 [hep-ph]

37. A. Rajaraman, Y. Shirman, J. Smidt, F. Yu, Phys. Lett. B 678, 367 (2009). doi:10.1016/j.physletb.2009.06.047. arXiv:0903.0668 [hep-ph]

38. A.M. Thalapillil, JHEP 1106, 059 (2011). doi:10.1007/ JHEP06(2011)059. arXiv:1012.4829 [hep-ph]

39. Y. Kats, P. Meade, M. Reece, D. Shih, JHEP 1202, 115 (2012). doi:10.1007/JHEP02(2012)115. arXiv:1110.6444 [hep-ph]

40. J.T. Ruderman, D. Shih, JHEP 1208, 159 (2012). doi:10.1007/ JHEP08(2012)159. arXiv:1103.6083 [hep-ph]

41. W. Porod, Comput. Phys. Commun. 153, 275 (2003). doi:10.1016/ S0010-4655(03)00222-4. arXiv:hep-ph/0301101 
42. W. Porod, F. Staub, Comput. Phys. Commun. 183, 2458 (2012). doi:10.1016/j.cpc.2012.05.021. arXiv:1104.1573 [hep-ph]

43. M. Dine, N. Seiberg, S. Thomas, Phys. Rev. D 76, 095004 (2007). doi:10.1103/PhysRevD.76.095004. arXiv:0707.0005 [hep-ph]

44. T. Sjostrand, S. Mrenna, P.Z. Skands, Comput. Phys. Commun. 178, 852 (2008). doi:10.1016/j.cpc.2008.01.036. arXiv:0710.3820 [hep-ph]

45. W. Beenakker, M. Klasen, M. Kramer, T. Plehn, M. Spira, P.M. Zerwas, Phys. Rev. Lett. 83, 3780 (1999). doi:10.1103/PhysRevLett. 83.3780. arXiv:hep-ph/9906298

46. W. Beenakker, M. Klasen, M. Kramer, T. Plehn, M. Spira, P.M. Zerwas, Phys. Rev. Lett. 100, 029901 (2008). doi:10.1103/ PhysRevLett.100.029901. arXiv:hep-ph/9906298

47. M. Drees, H. Dreiner, D. Schmeier, J. Tattersall, J.S. Kim, Comput. Phys. Commun. 187, 227 (2014). doi:10.1016/j.cpc.2014.10.018. arXiv:1312.2591 [hep-ph]
48. J. Cao, L. Shang, J.M. Yang, Y. Zhang, JHEP 1506, 152 (2015). doi:10.1007/JHEP06(2015)152. arXiv:1504.0786 [hep-ph]

49. G. Aad et al. [ATLAS Collaboration], JHEP 1310, 130 (2013). doi:10.1007/JHEP10(2013)130. arXiv:1308.1841 [hep-ex]

50. G. Aad et al. [ATLAS Collaboration], JHEP 1401, 109 (2014). doi:10.1007/JHEP01(2014)109. arXiv:1308.1841 [hep-ex]

51. G. Aad et al. [ATLAS Collaboration], JHEP 1310, 189 (2013). doi:10.1007/JHEP10(2013)189. arXiv:1308.2631 [hep-ex] 